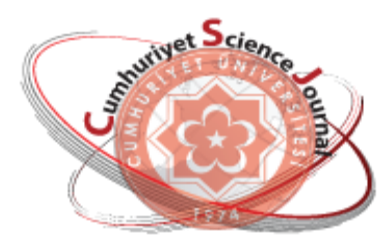

e-ISSN: 2587-246X

ISSN: $2587-2680$

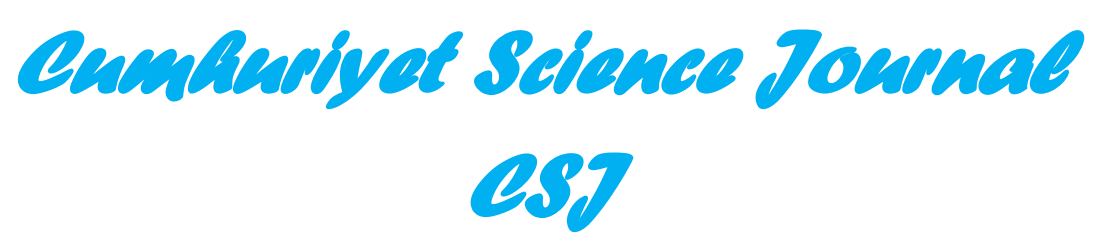

Cumhuriyet Sci. J., Vol.40-4 (2019) 902-908

\title{
Half-Inverse Problem For Dirac Operator With Boundary And Transmission Conditions Dependent Spectral Parameter Polynomially
}

\author{
Merve ARSLANTAŞ ${ }^{1}$ (D), Yalçın GÜLDÜ ${ }^{1 *}$ \\ ${ }^{1}$ Department of Mathematics, Faculty of Science, Cumhuriyet University 58140
}

Sivas, TURKEY

\begin{abstract}
In this paper, half-inverse problem is considered for Dirac equations with boundary and finite number of transmission conditions depending polynomially on the spectral parameter, if the potential is given over the half of the considered interval and if one spectrum is known then, potential function $\Omega(\mathrm{x})$ on the whole interval and the other coefficients of the considered problem can be determined uniquely.
\end{abstract}

Keywords: Dirac equations, Transmission conditions, Spectral parameter.

\section{Sınır ve Süreksizlik Koşulları Spektral Parametreye Polinom Olarak Bağlı Dirac Operatörü İçin Yarı-Ters Problem}

Özet. Bu makalede, sınır koşulları ve sonlu sayıda süreksizlik koşulları spektral parametreye polinom olarak bağlı Dirac denklemleri için yarı-ters problem ele alınmış olup, yarı aralıkta $\Omega(\mathrm{x})$ potansiyel fonksiyonu biliniyorken, bir spektruma göre, aralı̆ğın tamamında $\Omega(\mathrm{x})$ potansiyel fonksiyonu ile ele alınan problemin katsayılarının tek olarak belirlendiği gösterilmiştir.

Anahtar Kelimeler: Dirac denklemler, Süreksizlik koşulları, Spektral parametre.

\section{INTRODUCTION}

Inverse problems of spectral analysis compose of retrieving operators from their spectral characteristics. For this reason, inverse spectral theory is so significant research subject in mathematics, physics, mechanics, electronics, geophysics and other branches of natural sciences.

Half-inverse problem for a Dirac operator consists in reconstruction of the operator from its spectrum and known potential in the half-interval. Half inverse problem was first studied by Hochstadt and Lieberman in 1978[1]. In study of [2],by one boundary condition and potential which is known on half the interval, the potential and other boundary condition are uniquely determined. After that, these results have been used to lots of works[3-12].

On the other hand, in 1973 Walter [13] and in 1977 Fulton [14] studied the Sturm-Liouville problem with boundary conditions dependent on spectral parameter linearly. Then, inverse problems for some classes of differential operators depending on the eigenvalue-parameter linearly or nonlinearly on boundary and also transmission conditions were studied in various papers[15-33].

The main result of this paper is that if the potential function $\Omega(\mathrm{x})$ is known over the half the interval and one spectrum is given, then coefficients of the following problem can be uniquely determined. 
In this study, we concern the boundary value problem L generated by the following system of Dirac equations

$$
l[Y(x)]:=B Y^{\prime}(x)+\Omega(\mathrm{x}) \mathrm{Y}(\mathrm{x})=\lambda Y(x)
$$

with the boundary conditions

$$
\begin{aligned}
& l_{1} y:=a_{2}(\lambda) y_{2}(a)-a_{1}(\lambda) y_{1}(a)=0 \\
& l_{2} y:=b_{2}(\lambda) y_{2}(b)-b_{1}(\lambda) y_{1}(b)=0
\end{aligned}
$$

and the transmission conditions

$$
\begin{array}{lc}
U_{i}(y):= & y_{1}\left(\xi_{i}+0\right)-\theta_{i} y_{1}\left(\xi_{i}-0\right)=0 \\
V_{i}(y):= & y_{2}\left(\xi_{i}+0\right)-\theta_{i}^{-1} y_{2}\left(\xi_{i}-0\right)-\gamma_{i}(\lambda) y_{1}\left(\xi_{i}-0\right)=0
\end{array} i=\overline{1, n}
$$

where $B=\left(\begin{array}{cc}0 & 1 \\ -1 & 0\end{array}\right), \Omega(\mathrm{x})=\left(\begin{array}{cc}p(x) & q(x) \\ q(x) & r(x)\end{array}\right), Y(x)=\left(\begin{array}{l}y_{1}(x) \\ y_{2}(x)\end{array}\right), p(x), q(x)$ and $r(x)$ are real valued functions in $L_{2}(a, b), \lambda$ is a spectral parameter, $a_{i}(\lambda), b_{i}(\lambda),(i=1,2)$ and $\gamma_{i}(\lambda)(i=\overline{1, n})$ are polynomial with real cofficients and no common zeros, $\xi_{i} \in(a, b)(i=\overline{1, n}), \theta_{i} \in \mathbb{R}^{+}$,

$$
\begin{aligned}
& a_{1}(\lambda)=\sum_{k=0}^{m_{1}} a_{k 1} \lambda^{k}, a_{2}(\lambda)=\sum_{k=0}^{m_{2}} a_{k 2} \lambda^{k}, b_{1}(\lambda)=\sum_{k=0}^{m_{3}} b_{k 1} \lambda^{k}, b_{2}(\lambda)=\sum_{k=0}^{m_{4}} b_{k 2} \lambda^{k} \text { and } \\
& \gamma_{i}(\lambda)=\sum_{k=0}^{r_{i}} \gamma_{k i} \lambda^{k}, f(\lambda)=\frac{b_{1}(\lambda)}{b_{2}(\lambda)}, m_{a}=\max \left\{m_{1}, m_{2}\right\}, m_{b}=\max \left\{m_{3}, m_{4}\right\}, \\
& r=\max _{1 \leq i \leq n}\left\{\operatorname{deg} \gamma_{i}(\lambda)\right\} .
\end{aligned}
$$

\section{UNIQUENESS THEOREM}

\section{Theorem: Suppose}

$\lambda_{n}=\tilde{\lambda}_{n}, a_{i}(\lambda)=\tilde{a}_{i}(\lambda)(i=1,2), \Omega(\mathrm{x})=\widetilde{\Omega}(\mathrm{x})$ on $\left[a, \frac{a+b}{2}\right]$ and $U_{i}=\widetilde{U}_{i}, V_{i}=\tilde{V}_{i}$ for all $i=\overline{1, n}$ with $\xi_{i} \leq \frac{a+b}{2}$ for $\operatorname{deg} a_{2}(\lambda)>\operatorname{deg} a_{1}(\lambda)$

if $\operatorname{deg}_{2}(\lambda)>\operatorname{deg} b_{1}(\lambda) \quad m_{2}>m_{4}+\sum_{i=1}^{n} r_{i}$

if $\operatorname{deg} b_{1}(\lambda)>\operatorname{deg} b_{2}(\lambda) \quad m_{2}>m_{3}+\sum_{i=1}^{n} r_{i}$

if $\operatorname{deg} b_{1}(\lambda)=\operatorname{deg} b_{2}(\lambda) \quad m_{2}>m_{b}+\sum_{i=1}^{n} r_{i}$.

Then $\Omega(\mathrm{x})=\widetilde{\Omega}(\mathrm{x})$ almost everywhere on $[a, b], f(\lambda)=\tilde{f}(\lambda)$ and $\theta_{i}=\tilde{\theta}_{i}, \gamma_{i}(\lambda)=\tilde{\gamma}_{i}(\lambda), \xi_{i}=\tilde{\xi}_{i}$ for $i=\overline{1, n}$.

We need the following lemma, before we prove this theorem.

Lemma: If $\lambda^{*}$ is the zero of the polynomial $a_{2}(\lambda)$ with multiplicities $m_{\lambda^{*}}$ then $\lambda^{*}$ is also the zero of the entire function

$C \psi_{1}(a, \lambda)-\tilde{\psi}_{1}(a, \lambda)$

with at least multiplicities $m_{\lambda^{*}}$.

Proof: $\Delta(\lambda)=a_{1}(\lambda) \psi_{1}(a, \lambda)-a_{2}(\lambda) \psi_{2}(a, \lambda)$ and $\tilde{\Delta}(\lambda)=a_{1}(\lambda) \tilde{\psi}_{1}(a, \lambda)-a_{2}(\lambda) \tilde{\psi}_{2}(a, \lambda)$. 
If $\lambda^{*}$ is the zero of the polynomial $a_{2}(\lambda)$ with multiplicities $m_{\lambda^{*}}$ at that time we attain

$\Delta\left(\lambda^{*}\right)=a_{1}\left(\lambda^{*}\right) \psi_{1}\left(a, \lambda^{*}\right)$ and $\tilde{\Delta}\left(\lambda^{*}\right)=a_{1}\left(\lambda^{*}\right) \tilde{\psi}_{1}\left(a, \lambda^{*}\right)$.

On the other hand, since $\tilde{\Delta}(\lambda)$ and $\Delta(\lambda)$ are both entire in $\lambda$, by Hadamard's factorization theorem we can $\tilde{\Delta}(\lambda)=C \Delta(\lambda)$. Then

$0=C \Delta\left(\lambda^{*}\right)-\tilde{\Delta}\left(\lambda^{*}\right)=a_{1}\left(\lambda^{*}\right)\left[C \psi_{1}\left(a, \lambda^{*}\right)-\tilde{\psi}_{1}\left(a, \lambda^{*}\right)\right]$.

Since $a_{1}(\lambda)$ and $a_{2}(\lambda)$ do not have common zeros, we have

$C \psi_{1}\left(a, \lambda^{*}\right)-\tilde{\psi}_{1}\left(a, \lambda^{*}\right)=0$.

Now, inductively, if for all $0 \leq s<k \leq m_{\lambda^{*}}-1$ there holds

$\left.\frac{d^{s}}{d \lambda^{s}}\left[C \psi_{1}(a, \lambda)-\tilde{\psi}_{1}(a, \lambda)\right]\right|_{\lambda=\lambda^{*}}=0$

then we will prove that

$\left.\frac{d^{k}}{d \lambda^{k}}\left[C \psi_{1}(a, \lambda)-\tilde{\psi}_{1}(a, \lambda)\right]\right|_{\lambda=\lambda^{*}}=0$

$\frac{d^{k}}{d \lambda^{k}} \Delta(\lambda)=\frac{d^{k}}{d \lambda^{k}}\left[a_{1}(\lambda) \psi_{1}(a, \lambda)-a_{2}(\lambda) \psi_{2}(a, \lambda)\right]$

$=\sum_{t=0}^{k}\left(\begin{array}{c}k \\ t\end{array}\right)\left[\frac{d^{t}}{d \lambda^{t}} a_{1}(\lambda) \frac{d^{k-t}}{d \lambda^{k-t}} \psi_{1}(a, \lambda)-\frac{d^{t}}{d \lambda^{t}} a_{2}(\lambda) \frac{d^{k-t}}{d \lambda^{k-t}} \psi_{2}(a, \lambda)\right]$

$\left.\frac{d^{k}}{d \lambda^{k}} \Delta(\lambda)\right|_{\lambda=\lambda^{*}}=\left.\left.\sum_{t=0}^{k}\left(\begin{array}{c}k \\ t\end{array}\right) \frac{d^{t}}{d \lambda^{t}} a_{1}(\lambda)\right|_{\lambda=\lambda^{*}} \frac{d^{k-t}}{d \lambda^{k-t}} \psi_{1}(a, \lambda)\right|_{\lambda=\lambda^{*}}$

$\left.\frac{d^{k}}{d \lambda^{k}} \widetilde{\Delta}(\lambda)\right|_{\lambda=\lambda^{*}}=\left.\left.\sum_{t=0}^{k}\left(\begin{array}{c}k \\ t\end{array}\right) \frac{d^{t}}{d \lambda^{t}} a_{1}(\lambda)\right|_{\lambda=\lambda^{*}} \frac{d^{k-t}}{d \lambda^{k-t}} \tilde{\psi}_{1}(a, \lambda)\right|_{\lambda=\lambda^{*}}$

From the last two equations we have

$\left.\frac{d^{k}}{d \lambda^{k}}[C \Delta(\lambda)-\widetilde{\Delta}(\lambda)]\right|_{\lambda=\lambda^{*}}=\left.\left.\sum_{t=0}^{k}\left(\begin{array}{c}k \\ t\end{array}\right) \frac{d^{t}}{d \lambda^{t}} a_{1}(\lambda)\right|_{\lambda=\lambda^{*}} \frac{d^{k-t}}{d \lambda^{k-t}}\left[C \psi_{1}(a, \lambda)-\tilde{\psi}_{1}(a, \lambda)\right]\right|_{\lambda=\lambda^{*}}$

Since

$C \Delta(\lambda)-\tilde{\Delta}(\lambda)=0$

$\left.\left.\sum_{t=0}^{k}\left(\begin{array}{c}k \\ t\end{array}\right) \frac{d^{t}}{d \lambda^{t}} a_{1}(\lambda)\right|_{\lambda=\lambda^{*}} \frac{d^{k-t}}{d \lambda^{k-t}}\left[C \psi_{1}(a, \lambda)-\tilde{\psi}_{1}(a, \lambda)\right]\right|_{\lambda=\lambda^{*}}=0$.

Since for all $0 \leq s<k$

$\left.\frac{d^{s}}{d \lambda^{s}}\left[C \psi_{1}(a, \lambda)-\tilde{\psi}_{1}(a, \lambda)\right]\right|_{\lambda=\lambda^{*}}=0$

we have 
$\left.a_{1}\left(\lambda^{*}\right) \frac{d^{k}}{d \lambda^{k}}\left[C \psi_{1}(a, \lambda)-\tilde{\psi}_{1}(a, \lambda)\right]\right|_{\lambda=\lambda^{*}}=0$

Since $a_{1}\left(\lambda^{*}\right)$ we obtain

$\left.\frac{d^{k}}{d \lambda^{k}}\left[C \psi_{1}(a, \lambda)-\tilde{\psi}_{1}(a, \lambda)\right]\right|_{\lambda=\lambda^{*}}=0$

Lemma is proved.

If $a_{1}(\lambda) \equiv 0$ and $a_{2}(\lambda) \equiv 1$ are taken in the boundary condition, since $M(\lambda)=-\frac{\psi_{1}(a, \lambda)}{\Delta(\lambda)}$ and

$\Delta(\lambda)=a_{1}(\lambda) \psi_{1}(a, \lambda)-a_{2}(\lambda) \psi_{2}(a, \lambda)=-\psi_{2}(a, \lambda)$

$$
M_{0}(\lambda):=\frac{\psi_{1}(a, \lambda)}{\psi_{2}(a, \lambda)}
$$

obtained.

Remark 1: For the problem $L$ given by the conditions $a_{1}(\lambda) \equiv 0$ and $a_{2}(\lambda) \equiv 1$ if $M_{0}(\lambda)=\widetilde{M}_{0}(\lambda)$ then $\Omega(\mathrm{x})=\widetilde{\Omega}(\mathrm{x})$ almost everywhere on $[a, b], f(\lambda)=\tilde{f}(\lambda)$ and for each $i=\overline{1, n} \theta_{i}=\tilde{\theta}_{i}$, $\gamma_{i}(\lambda)=\tilde{\gamma}_{i}(\lambda), \xi_{i}=\tilde{\xi}_{i}$ applies.

Remark 2: For the Dirac operator given in the interval $\left[\frac{a+b}{2}, b\right]$ with equation (1), $y_{2}\left(\frac{a+b}{2}\right)=0$ and the transmission conditions (4), from the above Remark $1 ; M_{0}(\lambda)$ determines the function $\Omega(\mathrm{x})$ and $\theta_{i}$, $\gamma_{i}(\lambda), \xi_{i}$ for $i=\overline{1, n}$ as one on $\left[\frac{a+b}{2}, b\right]$.

Proof of Theorem : We only prove the case when $\xi_{i} \neq \frac{a+b}{2}$ for all $i$. The argument of the case when $\xi_{i}=\frac{a+b}{2}$ for some $i$ is similar. Using Lagrange identity we have

$$
\begin{aligned}
& \int_{a}^{\frac{a+b}{2}}\left(\tilde{\psi}_{1}(x, \lambda), \tilde{\psi}_{2}(x, \lambda)\right)(\Omega(\mathrm{x})-\widetilde{\Omega}(\mathrm{x}))\left(\begin{array}{l}
\psi_{1}(x, \lambda) \\
\psi_{2}(x, \lambda)
\end{array}\right) d x \\
& +\left.\left[\tilde{\psi}_{1}(x, \lambda) \psi_{2}(x, \lambda)-\tilde{\psi}_{2}(x, \lambda) \psi_{1}(x, \lambda)\right]\right|_{a} ^{\frac{a+b}{2}}
\end{aligned}
$$

For all $i$ satisfying $\xi_{i}<\frac{a+b}{2}$

$\left.\left[\tilde{\psi}_{1}(x, \lambda) \psi_{2}(x, \lambda)-\tilde{\psi}_{2}(x, \lambda) \psi_{1}(x, \lambda)\right]\right|_{\xi_{i}-0} ^{\xi_{i}+0}=0$

Together with $\Omega(\mathrm{x})=\widetilde{\Omega}(\mathrm{x})$ for a.e. $\left[a, \frac{a+b}{2}\right]$ we have

$\tilde{\psi}_{1}\left(\frac{a+b}{2}, \lambda\right) \psi_{2}\left(\frac{a+b}{2}, \lambda\right)-\tilde{\psi}_{2}\left(\frac{a+b}{2}, \lambda\right) \psi_{1}\left(\frac{a+b}{2}, \lambda\right)$

$=\tilde{\psi}_{1}(a, \lambda) \psi_{2}(a, \lambda)-\tilde{\psi}_{2}(a, \lambda) \psi_{1}(a, \lambda)$.

Since 
$\tilde{\Delta}(\lambda)=C \Delta(\lambda), \Delta(\lambda)=a_{1}(\lambda) \psi_{1}(a, \lambda)-a_{2}(\lambda) \psi_{2}(a, \lambda)$ and $\tilde{\Delta}(\lambda)=a_{1}(\lambda) \tilde{\psi}_{1}(a, \lambda)-a_{2}(\lambda) \tilde{\psi}_{2}(a, \lambda)$

we get

$\tilde{\psi}_{1}(a, \lambda) \psi_{2}(a, \lambda)-\tilde{\psi}_{2}(a, \lambda) \psi_{1}(a, \lambda)=\Delta(\lambda) \frac{C \psi_{1}(a, \lambda)-\tilde{\psi}_{1}(a, \lambda)}{a_{2}(\lambda)}$

Denote

$\mathrm{T}(\lambda):=\frac{\mathrm{G}(\lambda)}{\Delta(\lambda)}$

$=\frac{\tilde{\psi}_{1}\left(\frac{a+b}{2}, \lambda\right) \psi_{2}\left(\frac{a+b}{2}, \lambda\right)-\tilde{\psi}_{2}\left(\frac{a+b}{2}, \lambda\right) \psi_{1}\left(\frac{a+b}{2}, \lambda\right)}{\Delta(\lambda)}$

The function $\mathrm{T}(\lambda)$ is entire in $\mathbb{C}$. From the assumption that $\lambda_{n}=\tilde{\lambda}_{n}$ and the term of the characteristic function $\Delta(\lambda)$ it is easy to infer that

if $\operatorname{deg} b_{2}(\lambda)>\operatorname{deg} b_{1}(\lambda) \quad m_{4}=\widetilde{m}_{4}$

if $\operatorname{deg} b_{1}(\lambda)>\operatorname{deg} b_{2}(\lambda) \quad m_{3}=\widetilde{m}_{3}$

if $\operatorname{deg} b_{1}(\lambda)=\operatorname{deg} b_{2}(\lambda) \quad m_{b}=\widetilde{m}_{b}$.

From the following inequalities

$|\Delta(\lambda)| \geq C_{\delta}|\lambda|^{m_{2}+m_{4}+A} \exp \{|\operatorname{Im} \lambda|(b-a)\}, \quad \operatorname{deg} b_{2}(\lambda)>\operatorname{deg} b_{1}(\lambda)$

$|\Delta(\lambda)| \geq C_{\delta}|\lambda|^{m_{2}+m_{3}+A} \exp \{|\operatorname{Im} \lambda|(b-a)\}, \quad \operatorname{deg} b_{1}(\lambda)>\operatorname{deg} b_{2}(\lambda)$

$|\Delta(\lambda)| \geq C_{\delta}|\lambda|^{m_{2}+m_{b}+A} \exp \{|\operatorname{Im} \lambda|(b-a)\}, \quad \operatorname{deg} b_{1}(\lambda)=\operatorname{deg} b_{2}(\lambda)$

and the asymptotic formulas of the functions $\psi_{i}(x, \lambda),(i=1,2)$ for all intervals $\left(\xi_{i}, \xi_{i+1}\right),(i=\overline{0, n})$ and Phragmen-Lindelöf theorem, for all $\lambda$ we get $\mathrm{T}(\lambda) \equiv 0$.

Because of $G(\lambda) \equiv 0$ it yields

$\tilde{\psi}_{1}\left(\frac{a+b}{2}, \lambda\right) \psi_{2}\left(\frac{a+b}{2}, \lambda\right)-\tilde{\psi}_{2}\left(\frac{a+b}{2}, \lambda\right) \psi_{1}\left(\frac{a+b}{2}, \lambda\right)=0$

which is equivalent to

$\frac{\psi_{1}\left(\frac{a+b}{2}, \lambda\right)}{\psi_{2}\left(\frac{a+b}{2}, \lambda\right)}=\frac{\tilde{\psi}_{1}\left(\frac{a+b}{2}, \lambda\right)}{\tilde{\psi}_{2}\left(\frac{a+b}{2}, \lambda\right)}$.

From Remark 1 and Remark 2, proof of this theorem is finished.

Acknowledgement: The work was supported by grants from CUBAP (F-543).

\section{REFERENCES}

[1] Hochstadt, H., Lieberman, B., An inverse Surm-Liouville Problem with mixed given data, Siam J. Appl. Math., 34 -4 (1978) 676-680. 
[2] Hald, O. H., Discontiuous inverse eigenvalue problems, Comm. Pure Appl. Math., 37 (1984) 539577.

[3] Buterin, S., On half inverse problem for differential pencils with the spectral parameter in boundary conditions, Tamkang J. Math. , 42 (2011) 355-364.

[4] Özkan, A. S., Half inverse problem for a class of differential operator with eigenvalue dependent boundary and jump conditions, Journal of Advanced Research in Applied Mathematics, 4 (2011) 4349.

[5] Özkan, A. S., Half-inverse Sturm-Liouville problem with boundary and discontinuity conditions dependent on the spectral parameter, Inverse Problems in Science and Engineering. $22-5$ (2013) 848859.

[6] Sakhnovich, L., Half inverse problems on the finite interval, Inverse Probl., 17 (2001) 527-532.

[7] Wang, YP., Inverse problems for Sturm-Liouville operators with interior discontinuities and boundary conditions dependent on the spectral parameter, Math. Methods Appl. Sci., 36 (2013) 857-868.

[8] Yang, C-Fu., Uniqueness theorems for differential pencils with eigenparameter boundary conditions and transmission conditions, 255 (2013) 2615-2635.

[9] Yang, C-Fu., Inverse problems for Dirac equations polynomially depending on the spectral parameter, Applicable Analysis, (2015).

[10] Yang, C-Fu. (2011). Hochstadt-Lieberman theorem for Dirac operator with eigenparameter dependent boundary conditions, Nonlinear Anal. Ser. A: Theory Methods Appl., 74 (2011) 24752484 .

[11] Yang, C-Fu., Determination of Dirac operator with eigenparameter dependent boundary conditions from interior spectral data, Inv. Probl. Sci. Eng., 20 (2012) 351-369.

[12] Yang, C-Fu, Huang Z-You., A half-inverse problem with eigenparameter dependent boundary conditions, Numer. Func. Anal. Opt. , 31 (2010) 754-762.

[13] Walter, J., Regular eigenvalue problems with eigenvalue parameter in the boundary conditions, Math Z., 133 (1973) 301-312.

[14] Fulton, C. T. (1977). Two-point boundary value problems with eigenvalue parameter contained in the boundary conditions, Proc. R. Soc. Edinburgh, A77 (1977) 293-308.

[15] Amirov, R. Kh.; Keskin, B.; Özkan, A. S., Direct and inverse problems for the Dirac operator with spectral parameter linearly contained in boundary condition., Ukrainian Math. J., 61-9 (2009) 13651379.

[16] Binding, P. A.; Browne, P. J.; Watson, B. A., Inverse spectral problems for Sturm--Liouville equations with eigenparameter dependent boundary conditions, J. London Math. Soc. 62 (2000) 161-182 .

[17] Binding, P. A.; Browne, P. J.; Watson, B. A., Equivalence of inverse Sturm--Liouville problems with boundary conditions rationally dependent on the eigenparameter, J. Math. Anal. Appl. 291 (2004) 246-261.

[18] Binding, P. A.; Browne, P. J.; Seddighi, Sturm-Liouville problems with eigenparameter dependent boundary conditions, Proc. Edinb. Math. Soc. 37-2 (1993) 57-72.

[19] Browne, P. J.; Sleeman, B. D., A uniqueness theorem for inverse eigenparameter dependent Sturm-Liouville problems, Inverse Problem, 13 (1997) 1453-1462. 
[20] Chernozhukova, A.; Freiling, G., A uniqueness theorem for the boundary value problems with nonlinear dependence on the spectral parameter in the boundary conditions, Inverse Problems in Science and Engineering, 17-6 (2009) 777-785.

[21] Freiling, G.; Yurko, V. A., Inverse problems for Sturm-Liouville equations with boundary conditions polynomially dependent on the spectral parameter. Inverse Problems 26-5 (2010) 17.

[22] Gasymov, M. G.; Levitan, B. M., The Inverse Problem for the Dirac System, Dokl. Akad. Nauk SSR, 167 (1966) 967-970.

[23] Gasymov, M. G., The inverse scattering problem for a system of Dirac equations of order 2n. Dokl. Akad. Nauk SSSR 169 (1966) 1037-1040 (Russian); 11 676-678.

[24] Gasymov, M. G.; Dzhabiev, T. T., On the Determination of the Dirac System from Two Spectra, Transactions of the Summer School on Spectral Theory Operator, Baku/ELM., (1975) 46-71.

[25] Guliyev, N. J., Inverse eigenvalue problems for Sturm-Liouville equations with spectral parameter linearly contained in one of the boundary conditions. Inverse Problems 21-4 (2005) 1315-1330.

[26] Guseinov, IM., On the representation of Jost solutions of a system of Dirac differential equations with discontinuous coefficients, Izv. Akad. Nauk Azerb. SSR., 5 (1999) 41-45.

[27] Güldü, Y., On discontinuous Dirac operator with eigenparameter dependent boundary and two transmission conditions. Bound. Value Probl., (2016) 135- 19.

[28] Keskin, B., Inverse spectral problems for impulsive Dirac operators with spectral parameters contained in the boundary and discontinuity conditions polynomially, Neural Comput\&Applic. ,23 (2013) 1329-1333.

[29] Keskin, B., Inverse problems for impulsive Dirac operators with spectral parameters contained in the boundary and multitransfer conditions, Math. Methods in Applied Sciences, (2013) 38.

[30] Mennicken, R.; Schmid, H., Shkalikov, A. A., On the eigenvalue accumulation of Sturm-Liouville problems depending nonlinearly on the spectral parameter, Math. Nachr., 189 (1998) 157-170.

[31] Schmid, H.; Tretter, C., Singular Dirac systems and Sturm-Liouville problems nonlinear in the spectral parameter, J. Differ. Eqns., 181-2 (2002) 511-542.

[32] Yurko, V. A., Boundary value problems with a parameter in the boundary conditions, Izv. Akad. Nauk Armyan. SSR, Ser. Mat., 19-5 (1984) 398-409.

[33] Yurko, V. A. (2000). An inverse problem for pencils of differential operators, Mat Sbornik, 191-10 (2000) 137-158. 